\title{
High-Resolution Propagation Measurements using Biconical Antennas and Signal Processing
}

\author{
Robert T. Johnk ${ }^{1}$, John D. Ewan, Nicholas DeMinco, Ronald L. Carey, Paul McKenna, Christopher J. Behm, \\ Timothy J. Riley, Steven Carroll, Mark A. McFarland, James W. Leslie \\ Institute for Telecommunication Sciences \\ 325 Broadway \\ Boulder, Colorado 80305 USA \\ bjohnkeits.bldrdoc.gov
}

\begin{abstract}
This paper describes how commercially-available EMC biconical antennas can be used to perform high-resolution propagation measurements. A measurement procedure and signal processing sequence is described that greatly improves the range resolution and fidelity of transmission measurements using a pair of biconical antennas. Measurement results are provided for a number of different scenarios both indoors and outdoors. Direct comparisons are also provided with the Numerical Electromagnetics Code (NEC). The results obtained so far look quite promising and demonstrate the viability of the approach.
\end{abstract}

\section{INTRODUCTION}

Engineers at the Institute for Telecommunication Sciences (NTIA/ITS) are investigating the use of commercial-off-theshelf (COTS) antennas to perform high-resolution propagation measurements. The objective of this research is to provide the EMC and wireless measurements communities a useful tool for radiated test site validations and Radio-Frequency (RF) propagation measurements. COTS EMC antennas typically do not have impulse response characteristics that are suitable for high-resolution propagation measurements in the time domain An earlier numerical study showed that impulse response durations of dipole, biconical, and log-periodic antennas can be significantly reduced using the combination of a two-step measurement procedure and deconvolution $[1,2]$.

Experiments and numerical modelling are being conducted to see if this method can be practically implemented using a vector network analyzer (VNA) and modest antenna setups. An extensive series of experiments has been conducted using COTS biconical antennas in both indoor and outdoor environments. Transmission measurements have been performed outdoors over a metal ground plane, asphalt, and bare ground. Measurements have also been carried out in a fully-anechoic chamber.

Numerical models of selected biconical antenna deployments have also been developed using the Numerical Electromagnetics Code (NEC-2) [3]. The numerical simulations closely approximate the antenna deployments and data acquisition modes. The measured and simulated data can be directly intercompared as a benchmark.

The biconical antenna results look promising. A five-fold reduction in the impulse response time is realized using deconvolution, yielding sharp and clearly defined waveforms. The agreement between the numerical simulations and the measurements is good with typical discrepancies in the $1-2$
$\mathrm{dB}$ range. Additionally, the antenna mounting requirements are modest and can be implemented using low-cost dielectric carts and masts.

\section{MEASUREMENT SYSTEM}

The system used to perform the transmission measurements is shown in Fig. 1. It consists of a vector network analyzer, analog optical link, transmit and receive antennas, and interconnecting RF cables. The two antennas are two nominally identical biconical antennas used for low-frequency EMC radiated emissions testing. The specified frequency range for these antennas is $20-300 \mathrm{MHz}$. The transmit port of the VNA is connected to the transmit antenna through a precision $50-\mathrm{Ohm}$ coaxial cable. The receive antenna is connected to the optical transmitter with coaxial cable, which up-converts the applied RF signal to optical frequencies. When a calibration is applied to the system, it preserves the phase and amplitude of the received RF signal. The upconverted signal is then transmitted through a single-mode optical fiber cable to the optical receiver, which downconverts the signal at the same RF frequency. The advantages of the optical link are low loss (typically $0.1 \mathrm{~dB} / \mathrm{km}$ ) and low noise. Measurements have been performed with antenna separations as large as $250 \mathrm{~m}$. The heart of this measurement system is the VNA, which combines a transmitter and receiver in one box and is capable of sweeping over a large frequency range $(300 \mathrm{kHz}-6 \mathrm{GHz})$.

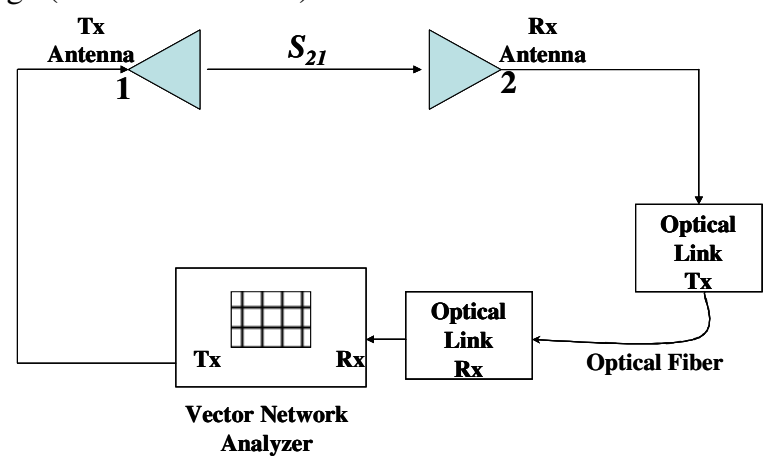

Fig. 1. Transmission measurement system.

The system is calibrated by connecting the receive antenna RF cable (point 2 in Fig.1) directly to the transmit antenna RF cable (point 1) using a $30 \mathrm{~dB}$ attenuator to avoid overdriving 


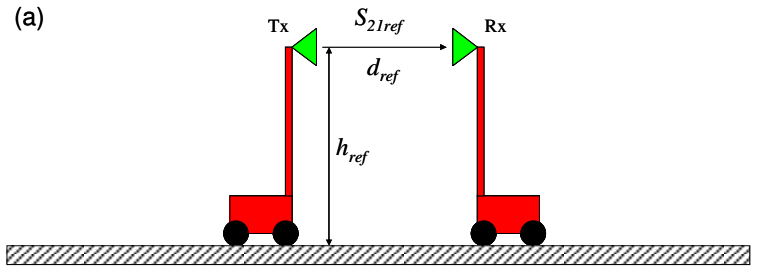

(b)

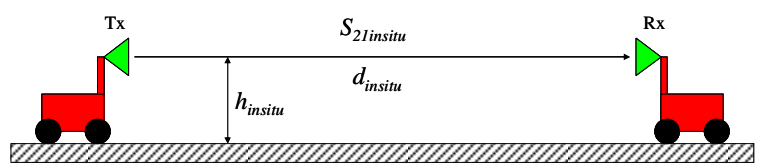

Fig. 2. Measurement sequence. (a) Free-space reference. (b) In-situ transmission measurement.

the link. A standard thru calibration is then performed to remove the effects of the link and cables, and it provides a reference at the antenna connector ports. The system measures the magnitude and phase of the transmission S-parameter $S_{21}$.

\section{MEASUREMENT SEQUENCES}

The process consists of two distinct and separate measurements. The first is the reference transmission measurement, shown in Figs. $2 \mathrm{a}$ and $3 \mathrm{a}$. The purpose of this measurement is to approximate a free-space condition. The antennas must be positioned well above ground and time gating must be applied to eliminate reflections from ground and nearby objects. The antennas are raised to a height of $h_{r e f}$
$=7 \mathrm{~m}$, with a separation of $d_{\text {ref }}=3 \mathrm{~m}$. The antennas are attached to and mounted on dielectric carts with extendable fiberglass masts to minimize spurious reflections. The biconicals are horizontally polarized to minimize interactions between the antennas and the attached coaxial cables. The second configuration, shown in Figs. $2 b$ and $3 b$, is the in-situ transmission measurement. This is the primary measurement we wish to enhance. In this case, the antennas are typically situated close to nearby objects and ground. The antennas are separated at a distance $d_{\text {insitu }}$ and set at a height of $h_{\text {insitu }}$. The choice of antenna heights and separation are dictated by the measurement scenario under consideration. They are typically different (but not always) from those of the reference measurement. The range of heights and separations that we used were $0.5 \mathrm{~m} \leq h_{\text {insitu }} \leq 8 \mathrm{~m}$ and $1 \mathrm{~m} \leq d_{\text {insitu }} \leq 250 \mathrm{~m}$.

\section{SignAL PROCESSING}

The goal of signal processing is to improve signal fidelity and range resolution by post processing of the in-situ and reference data to obtain useful time- and frequency-domain parameters. Parameters of interest might include path loss and delay spread [4]. The signal processing uses an efficient sequence of filtering, gating, time/frequency transformations, and deconvolution to mitigate these effects.

The post-processing consists of two elements: filtering and gating. Filtering conditions the signal and eliminates out-ofband effects. Gating is used to isolate desired transmission events. The goal is to obtain selected time- and frequencydomain quantities with minimized spurious effects. (a)

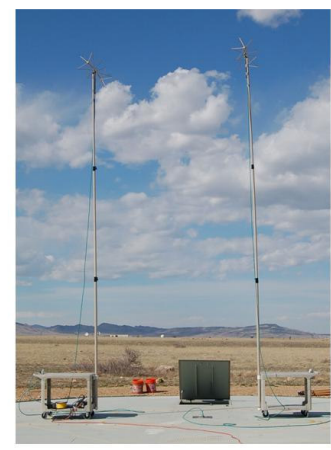

(b)

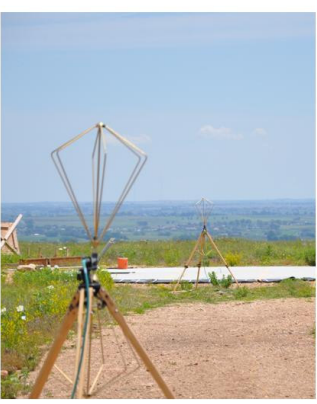

Fig. 3. (a) Free-space reference with horizontally-polarized biconical with $h=7 \mathrm{~m}$ and $d=3 \mathrm{~m}$. (b) An example of an in-situ setup with $h=$ $1 \mathrm{~m}$, and $d=30 \mathrm{~m}$.

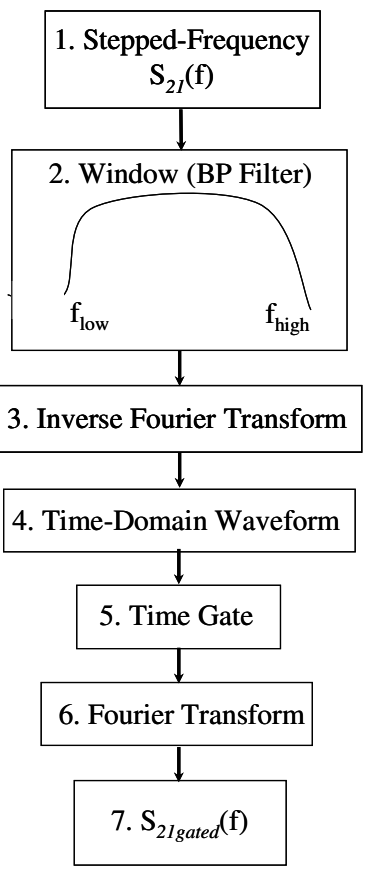

Fig. 4.Signal processing sequence. 


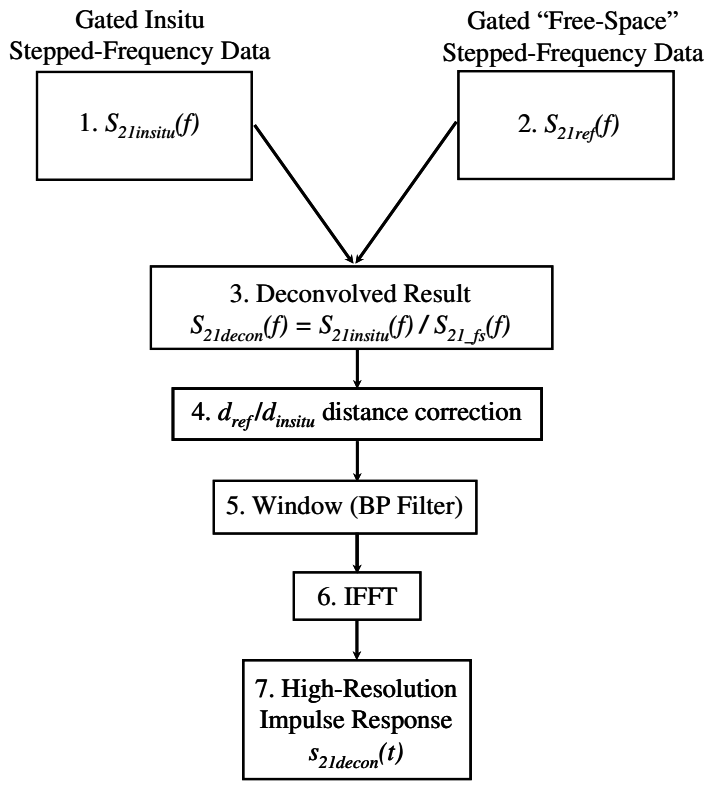

Fig. 5. The Process of Deconvolution.

Initially, the in-situ and reference data are filtered and gated using the steps shown in Fig. 4. Filtering conditions the signal by reducing out-of-band system noise and RF ambient energy, and it mitigates systematic out-of-band effects. Gating enhances the desired transmission events and suppresses undesired scattering and radio interference that are not of interest to a particular measurement. This improves measurement fidelity.

The stepped-frequency S-parameter data are bandpass filtered, both to eliminate low-frequency effects below the low-frequency cutoff of the optical link, and to suppress signal components above the $300 \mathrm{MHz}$ upper limit of the biconical antennas. The filtering is realized by applying a Kaiser-Bessel window [5]. The data are next inverse Fourier transformed to obtain a real-value, time-domain waveform. The waveform contains a combination of direct antenna-to-antenna coupling, ground reflections, and other scattering events. A rectangular time gate is then applied. The components within the gate are not altered, and the components outside are set to zero. The gated waveform is Fourier transformed to obtain the gated transmission $S_{21 \text { gated }}$.

The filtered and gated in-situ and reference measurements are further processed using deconvolution [2]. The signal processing sequence consists of seven steps as is shown in Fig. 5. First, the frequency-domain in-situ data are divided by the corresponding gated references, resulting in a normalized spectrum. Since the reference and in-situ measurements are often performed at different antenna separations, a $1 / r$ distance correction factor is applied to re-scale reference data to the in-situ separation. A bandpass filter is used to condition the data and remove spurious out-of-band responses. In the final step, an inverse Fourier transform is applied, and the result is a time-domain waveform with higher range resolution. This occurs because the antenna impulse response is removed by the initial normalization step. (a)

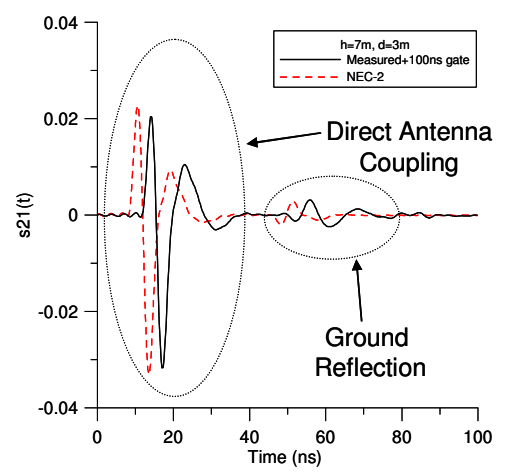

(b)

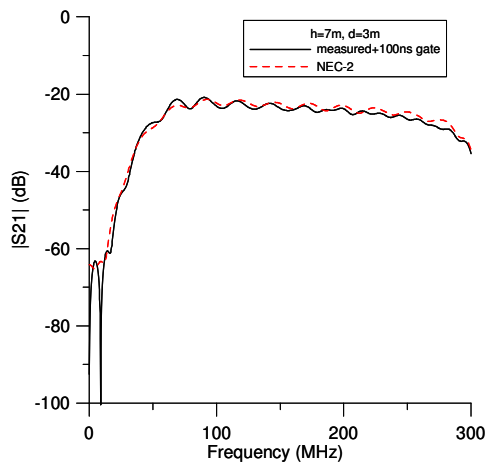

Fig. 6. (a) Measured (black) and computed (red) waveforms (step 5 of Fig. 4) for $h=7 \mathrm{~m}, d=3 \mathrm{~m}$. (b) Measured (black) and computed (red) amplitude spectra (step 7 of Fig. 4). A time gate with an interval of $0-100 \mathrm{nsec}$ was applied to both measured and simulated results.

\section{FREE-SPACE ReFERENCE RESUlts}

A series of measurements were performed using the test setup of Fig. 3a with two horizontally-polarized biconical antennas with $d=3 \mathrm{~m}$ and $h=7 \mathrm{~m}$. The antennas have the standard wire basket geometry and use 4:1 baluns for impedance matching. Stepped-frequency measurements were performed over the range of $300 \mathrm{kHz}-300 \mathrm{MHz}$, in $300 \mathrm{kHz}$ steps. A NEC-2 model of this test setup was also developed for comparison. The model was configured to simulate the stepped-frequency measurements, the test setup geometry, and effects of ground. The ground constants were assumed to be $\left(\varepsilon_{r}=5, \sigma=0.01 \mathrm{~S} / \mathrm{m}\right)$. Baluns were not incorporated into the model. In addition, a NEC-2 model was developed for the two biconicals at a separation of $d=3 \mathrm{~m}$ in a free-space environment without a ground. This model allows the computation of the free-space S-parameters without any processing, and it provides a benchmark to assess the impact of signal processing.

Figures $6 \mathrm{a}$ and $6 \mathrm{~b}$ show both measured and modelled timeand frequency-domain results obtained over ground with a time-gate interval of $0-100 \mathrm{~ns}$, and filter settings of $f_{\text {low }}=20$ $\mathrm{MHz}$, and $f_{\text {high }}=300 \mathrm{MHz}$. Good agreement is seen in the time-domain waveforms of Fig. 6a. The initial antenna-toantenna coupling exhibits a characteristic triplet structure with an impulse response duration of approximately $20-25 \mathrm{~ns}$, followed by ground reflection wavelet $35 \mathrm{~ns}$ later. There is an interval between the direct coupling and ground reflection 


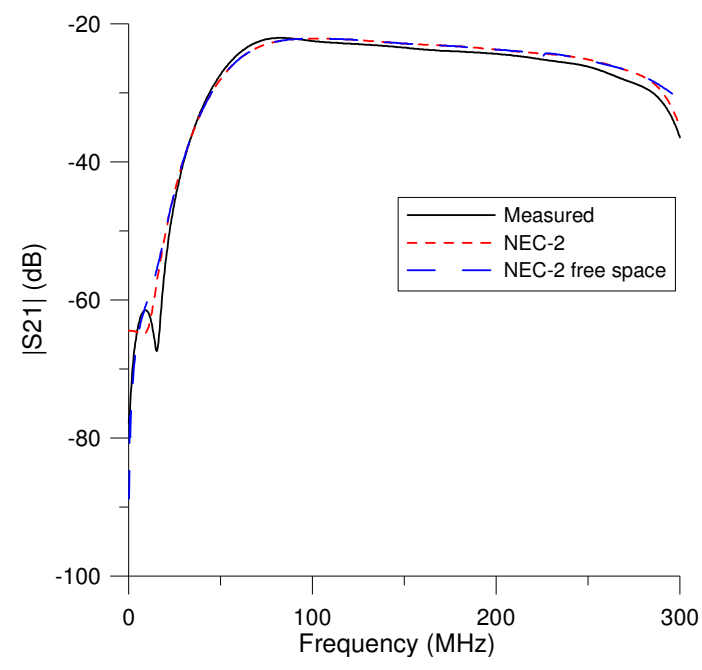

Fig. 7. Intercomparison of measured ( $45 \mathrm{~ns}$ gate), NEC-2 simulation (0-45 ns time gate), and NEC-2 free-space computed $S_{21}$ result (benchmark).

where the signal has minimal energy - a gate can be applied here. The modelled waveform occurs approximately $4 \mathrm{~ns}$ earlier than the measured one, since balun effects and an associated delay are not incorporated into the NEC model. Balun losses also account for slightly lower amplitudes. Good agreement $(1-3 \mathrm{~dB})$ is also seen between the corresponding frequency-domain amplitude spectra in Fig. 6b. The resulting spectra exhibit a characteristic scalloping that is due to the interference between the direct antenna coupling and the ground reflection.

A study of Fig. 6a indicates that a time gate of $0-45 \mathrm{~ns}$ passes only the direct antenna-to-antenna coupling and suppresses all subsequent scattering. NEC-2 simulations were also performed with the antennas at $d=3 \mathrm{~m}$ in an infinite, free-space environment. Fig. 7 shows measured results with a $0-45$ ns gate applied and two NEC simulations: one with the same $45 \mathrm{~ns}$ wide gate, and the free-space model with the computed s-parameters. Discrepancies of $1-2 \mathrm{~dB}$ are seen between the measurements and the two simulations $(0-45 \mathrm{~ns}$ gate) over most of the $20-300 \mathrm{MHz}$ frequency range. The free-space simulation does highlight the impact of gating in $280-300 \mathrm{MHz}$ range, where a downward shift is noted.

\section{IN-SITU RESULTS}

A series of in-situ measurements were performed on an asphalt plaza, located at the ITS Table Mountain Field Site, near Boulder, Colorado. Transmission was measured at two antenna heights and four separations using the two-cart configuration of Fig. 3a. The antennas were set at a height of either $7 \mathrm{~m}$ or $3.5 \mathrm{~m}$, and were separated at distances of $3,6,8$, and $10 \mathrm{~m}$.

Figure 8a shows the in-situ waveform for horizontallypolarized biconicals at $h=3.5 \mathrm{~m}$ and $d=3 \mathrm{~m}$. The waveform is almost identical to that of the free-space reference of Fig. $6 \mathrm{a}$ for times less than $25 \mathrm{~ns}$, after which, a ground reflection occurs. The ground reflection is not separable from the direct coupling in the in-situ waveform. The situation changes with (a)
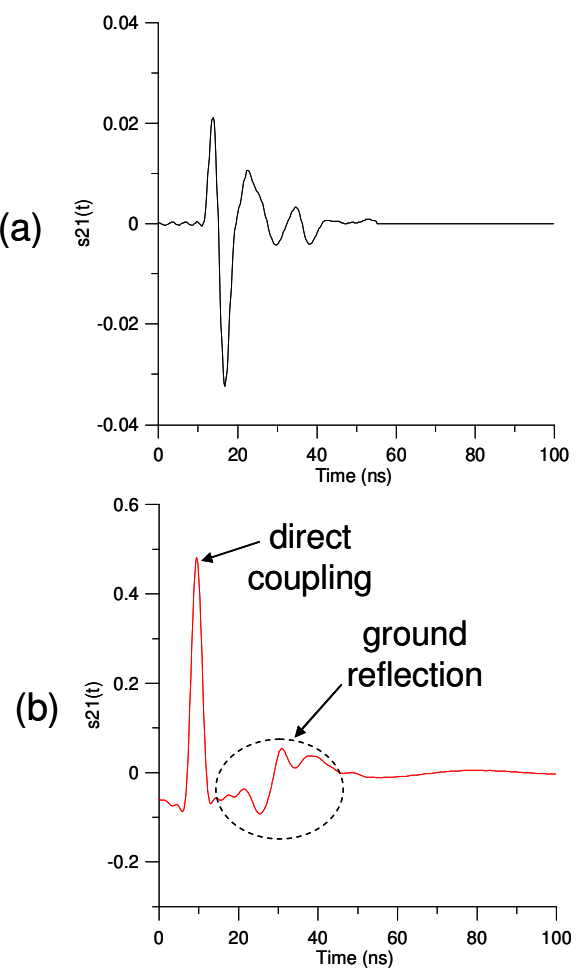

Fig. 8. (a) Measured in-situ waveform (step 5, Fig. 4) for $h=3.5 \mathrm{~m}$ and $d=3 \mathrm{~m}$ and horizontal polarization with a $0-55 \mathrm{~ns}$ gate. (b) Deconvolved waveform (step 7, Fig. 5) showing clear separation between the direct antenna coupling and the ground reflection.

(a)
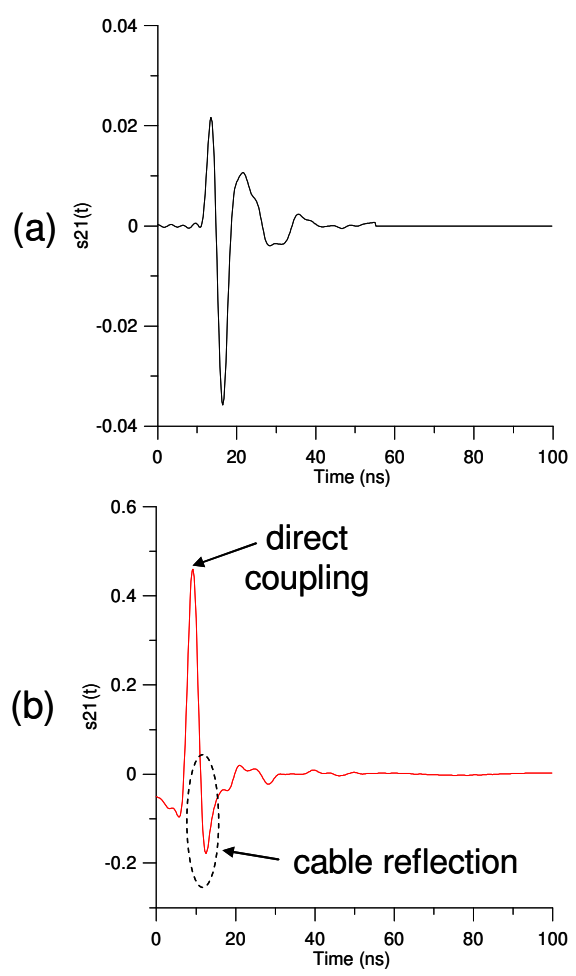

Fig. 9. (a) Measured in-situ waveform for $h=3.5 \mathrm{~m}$ and $d=3 \mathrm{~m}$ and vertical polarization. (b) Deconvolved waveform showing clear separation between the direct antenna coupling and cable reflections. 

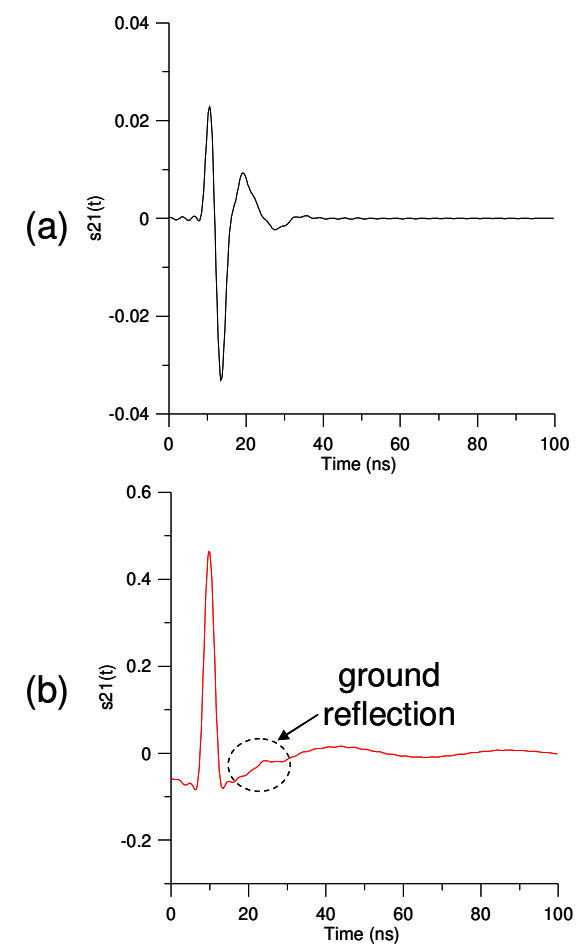

Fig. 10. (a) NEC-2 in-situ waveform for $h=3.5 \mathrm{~m}$ and $d=3 \mathrm{~m}$ and vertical polarization. (b) Deconvolved waveform showing a weak ground reflection. The assumed ground parameters are $\varepsilon_{\mathrm{r}}=5.0$ and $\sigma=0.01 \mathrm{~S} / \mathrm{m}$.

deconvolution, as is seen in Fig. 8b. The two waveform components are separable, and we see a doublet due to ground reflection.

When the antennas are moved to vertical polarization, the resulting in-situ waveform of Fig. 9a is different. The first negative peak that occurs at $18 \mathrm{~ns}$ is now larger than observed for horizontal polarization. Also, the rest of the waveform is different. Fig 9b shows the waveform after deconvolution is applied, and a sharp negative excursion is noted just after the direct coupling component. The $4 \mathrm{~ns}$ delay between these packets indicates that this is a reflection from the co-polarized feed cables, which are located $60 \mathrm{~cm}$ behind the transmit and receive antennas. It is interesting that this component is absent in the horizontally-polarized case for which the cables are cross-polarized to the antennas. The corresponding NEC-2 simulations for this configuration are shown in Figs. 10a and 10b. The NEC model assumes gap voltage source and does not incorporate cable effects. As a result, the sharp negative peak seen in the measurements is absent in the deconvolved NEC results. It is interesting to see that a weak ground reflection is visible in the deconvolved NEC results as well. This is due to the dipole-like pattern characteristics of the biconical antennas. A ground reflection is not distinctly visible in the measured results, since the strong cable reflections dominate.

\section{ANECHOIC CHAMBER RESUlTS}

Transmission measurements were performed using a pair of biconical antennas that were deployed inside a fully anechoic chamber, located at the National Institute of Standards and

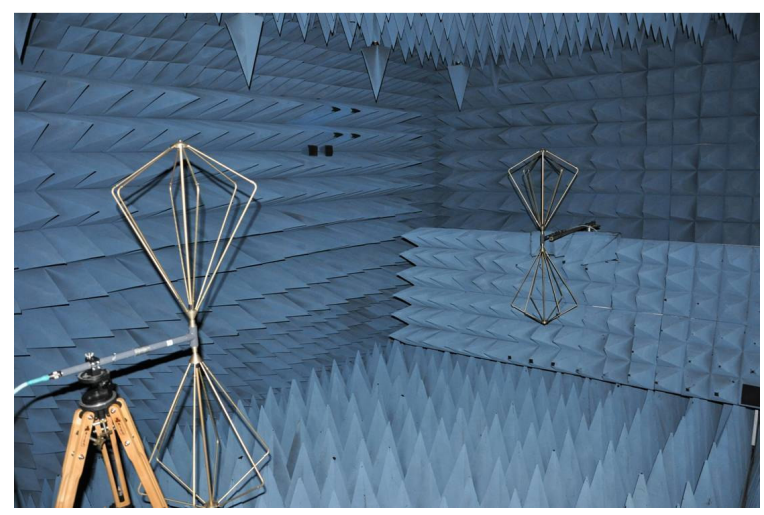

Fig. 11. Vertically-polarized biconical antennas inside an anechoic chamber.
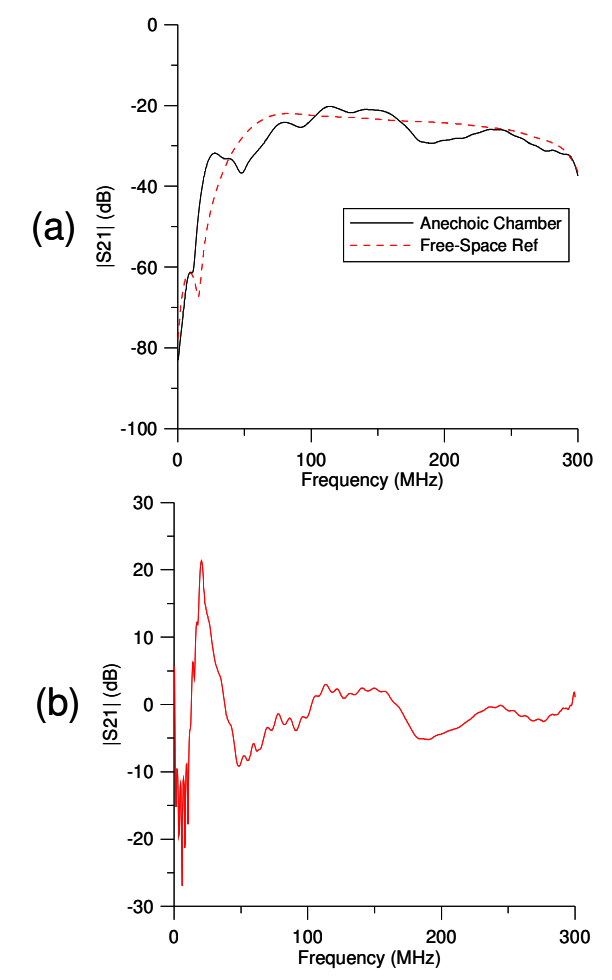

Fig. 12. (a) Transmission amplitude spectrum (step 7, Fig. 4) with vertically-polarized biconicals at a separation of $d=3 \mathrm{~m}$ inside an anechoic chamber $(0-400 \mathrm{~ns}$ gate) and a gated free-space reference (0-45 ns gate). (b) Normalized amplitude spectrum (step 3, Fig. 5). Note the strong cavity resonance at $18 \mathrm{MHz}$.

Technology (NIST) laboratory in Boulder, Colorado. The anechoic chamber, shown in Fig. 11, is a rectangular shaped room, with the dimensions $4.9 \mathrm{~m}$ high $\times 6.7 \mathrm{~m}$ wide $\times 8.5 \mathrm{~m}$ long [6]. The NIST chamber walls are covered with carbondoped pyramidal absorber, and it has an operational frequency range of $200 \mathrm{MHz}-40 \mathrm{GHz}$. Below $200 \mathrm{MHz}$ the absorber becomes increasingly reflective as the frequency decreases [7].

The biconical antennas were spaced at $d=3 \mathrm{~m}$, as is shown in Fig. 11, and measurements were performed for both vertical and horizontal polarization. Fig. 12a shows measured data obtained from the chamber and the gated free-space reference. 


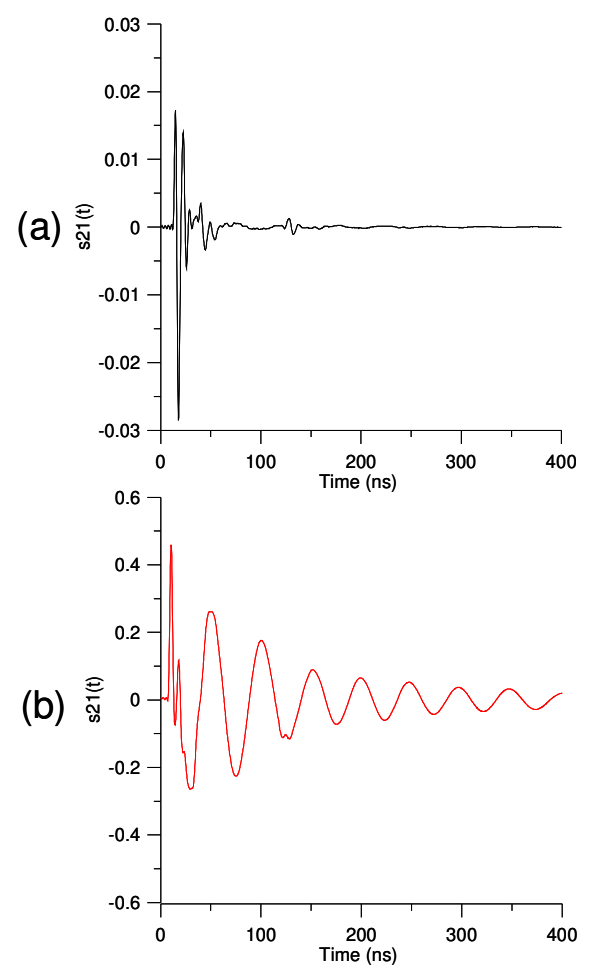

Fig. 13. (a) In-situ anechoic chamber waveform (step 5, Fig. 4) obtained with biconicals at a separation of $d=3 \mathrm{~m}$. (b) Deconvolved waveform (step 7, Fig. 5). The decaying sinusoidal waveform is the due to the $18 \mathrm{MHz}$ chamber resonance.

A study of the curves indicates that the chamber does have a significant influence over the entire frequency range. The deconvolved results that are shown in Fig. 12b highlight the impact of the chamber more dramatically. This curve is the difference between the free-space reference and the chamber, and provides a metric for the effectiveness of the installed absorber system [8]. The peak-to-peak deviations increase at frequencies below $200 \mathrm{MHz}$, reaching a maximum value of 22 $\mathrm{dB}$ at $18 \mathrm{MHz}$. This is caused by a longitudinal cavity resonance that occurs along the $8.5 \mathrm{~m}$ dimension of the chamber and the fact that the cones no longer provide much absorption. The corresponding in-situ and deconvolved timedomain data are plotted in Figs. 13a and 13b respectively.

The deconvolved waveform is particularly interesting, as it resolves the direct antenna coupling and early-time reflections from both the cables and absorbers, which are in close proximity to the receive antenna. The most interesting component is the decaying sinusoid that occurs approximately $10 \mathrm{~ns}$ after the onset of the waveform. This is caused by the strong $18 \mathrm{MHz}$ cavity resonance. Clearly, more insight is gained from the deconvolved waveform, as this resonance is not nearly as visible in the in-situ waveform.

\section{CONCLUSION}

This work demonstrates that high-resolution time-domain waveforms can be obtained using COTS biconical antennas with modest and simple test setups combined with straightforward measurement procedures. Deconvolution permits the resolution of scatterers for many typical configurations and greatly enhances waveform fidelity. One additional point that should be made is that using a VNA and calibrating the system to the antenna ports enables one to perform the reference and in-situ measurements at different times. For instance, reference measurements can be performed at one time, and in-situ measurements performed weeks, or even months later. This, of course, assumes that the antenna characteristics do not change between the measurements. All in all, this method is quite promising.

\section{ACKNOWLEDGEMENTS}

First and foremost the authors thank Wayde Allen of the ITS measurements division for his enthusiastic support of this research. The authors are also indebted to Randy Hoffman of the ITS measurements division for his excellent and constructive inputs to improve this paper. The authors also thank Frank Sanders, Eric Nelson, Patti Raush, and Jeff Bratcher of the ITS management team for their help and generous support. We also extend our heartfelt thanks to Dennis Camell of NIST for his outstanding support and help with the anechoic chamber measurements.

\section{REFERENCES}

[1] R. T. Johnk, "High-resolution time-domain site attenuation measurements using conventional emc test antennas-a numerical study," IEEE International Symposium on EMC, 18 to 22 August 2008, pp. 1 to 6

[2] N.S. Nahman and M.E. Guillaume, "Deconvolution of time-domain waveforms in the presence of noise," National Bureau of Standards (U.S.) Tech. Note 1047 (1981).

[3] Unofficial NEC-2 home page web address: http://www.NEC2.org/

[4] Saunders, S., and Aragón-Zavala, A., Antennas and Propagation for Wireless Communication Systems, $2^{\text {nd }}$ ed., New York, New York, John Wiley \& Sons, 2007.

[5] R.T. Johnk, B. Archambeault, D. Novotny, "Using joint timefrequency analysis to enhance time-domain numerical EMC simulations," Interference Technology, 2003.

[6] B. Fourestie, Z. Altman, M. Kanda, "Anechoic chamber evaluation using the matrix pencil method," IEEE Transactions on Electromagnetic Compatibility, vol. 41, no. 3, pp. 169 - 174, August, 1999.

[7] R. T. Johnk, A. R. Ondrejka, S. Tofani, and M. Kanda, "Time-domain measurements of the electromagnetic backscatter of pyramidal absorbers," IEEE Transactions on Electromagnetic Compatibility, vol. 35, no. 3, pp. 429 - 433, November, 1993.

[8] R. T. Johnk, D R. Novotny, C.M. Weil, M. Taylor, T.J. O'Hara, "Efficient and accurate testing of an EMC compliance chamber using an ultra-wideband measurement system," IEEE International Symposium on EMC, 13 to 17 August 2001, pp. 302 - 307. 